\title{
Erythrocyte-Bound Tissue Plasminogen Activator is Neuroprotective in Experimental Traumatic Brain Injury
}

\author{
Sherman C. Stein, Kumkum Ganguly, ${ }^{2}$ Caitlin M. Belfield, Xiangsheng Xu, Edward W. Swanson, \\ Xiao-Han Chen,' Kevin D. Browne,' Victoria E. Johnson,', Douglas H. Smith,' David G. LeBold, \\ Douglas B. Cines, ${ }^{3}$ and Vladimir R. Muzykhantov ${ }^{4}$
}

\begin{abstract}
The purpose of this study was to test the effects of exogenous tissue plasminogen activator (tPA) in traumatic brain injury (TBI). We tested two different tPA formulations, free tPA and tPA bound to erythrocytes (RBC/tPA). Vehicle and each of the tPA treatments were injected intravenously into anesthetized rats 15 min after moderate lateral fluid percussion injury. The animals were sacrificed at 2 days for calculating microclot burden $(n=13)$ and IgG staining area $(\mathrm{n}=13)$ in the brain sections as indicators of post-traumatic thrombosis and blood-brain barrier (BBB) breakdown, respectively. Another set of injured animals treated in the same way were sacrificed at 7 days to compare cortical lesion volumes $(n=28)$ and CA3 hippocampal cell loss $(n=24)$. All evaluations were done blinded with respect to treatment. No significant differences were found with respect to microclot burden or IgG staining volume. Injection of wild-type tPA caused significantly $(p<0.05)$ larger cortical injuries and greater cerebral hemorrhage. In contrast, there was significantly less cortical injury $(p<0.01)$ and hippocampal cell loss $(p<0.01)$ in the $\mathrm{RBC} / \mathrm{tPA}$ group than in all other groups. These results reveal that $\mathrm{RBC} / \mathrm{tPA}$ is more neuroprotective in experimental TBI than is unbound tPA.
\end{abstract}

Key words: blood-brain barrier; microclots; tissue plasminogen activator; traumatic brain injury

\section{Introduction}

$\mathbf{S}_{\mathrm{c}}^{\mathrm{z}}$ EVERAL inVESTIGATORS HAVE IDENTIFIED EVIDENCE of cerebral hypoxia-ischemia in patients with traumatic brain injury (TBI), as shown by autopsy (Graham and Adams, 1971; Graham et al., 1989), cerebral blood flow (Hlatky et al., 2004), and brain tissue oxygen level measurements (Stiefel et al., 2006). Although some forms of ischemia are the result of vascular compression, both the cause and clinical significance of other ischemia types are uncertain (Teasdale and Graham, 1998). Our laboratory and others have reported the presence of thrombi in microvessels of patients and laboratory animals following TBI (Lu et al., 2004; Stein et al., 2002; Stein et al., 2004) (Fig. 1). We have hypothesized that these microclots are the result of blood reacting with tissue factor, which is present in high concentrations on the adventitial surface of cerebral vessels (Fleck et al., 1990). This results in the intravascular clotting and formation of thrombi that occlude cerebral arterioles in the days following injury. This in turn may result in cerebral ischemia, with aggravation of the initial trauma (Stein and Smith, 2004). In theory, dissolving these thrombi might alleviate secondary damage and improve the outcome of TBI, if it could be done without impairing normal hemostasis and inflicting other adverse effects on the CNS.

Tissue plasminogen activator (tPA) is a potent thrombolytic agent that has been used with success for emergency treatment of ischemic stroke. Although its use might ameliorate damage done by clots, there are several drawbacks to tPA administration. An obvious problem is the likelihood that tPA indiscriminately dissolves both pathological intravascular microclots and mural hemostatic plugs, thereby worsening post-traumatic hemorrhage. Furthermore, the pharmacokinetics of tPA seem to be unsuitable for prevention of secondary post-traumatic clots. Intravascular microclot formation, development, and dissolution occur over days (Lu et al., 2004). Unfortunately, exogenous tPA is cleared from the circulation within minutes (Narita et al., 1995), thus limiting its therapeutic utility in this setting.

\footnotetext{
${ }^{1}$ Department of Neurosurgery, ${ }^{3}$ Department of Pathology and Laboratory Medicine, and ${ }^{4}$ Institute for Environmental Medicine, Institute for Translational Medicine and Department of Pharmacology, University of Pennsylvania School of Medicine, Philadelphia, Pennsylvania.

${ }^{2} \mathrm{~B}$ Division, Los Alamos National Laboratory, Los Alamos, New Mexico.
} 
a

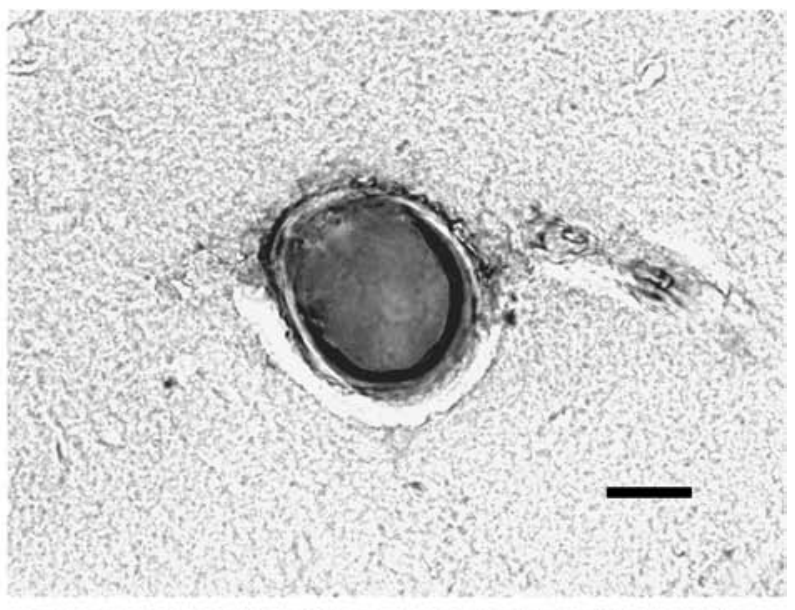

b

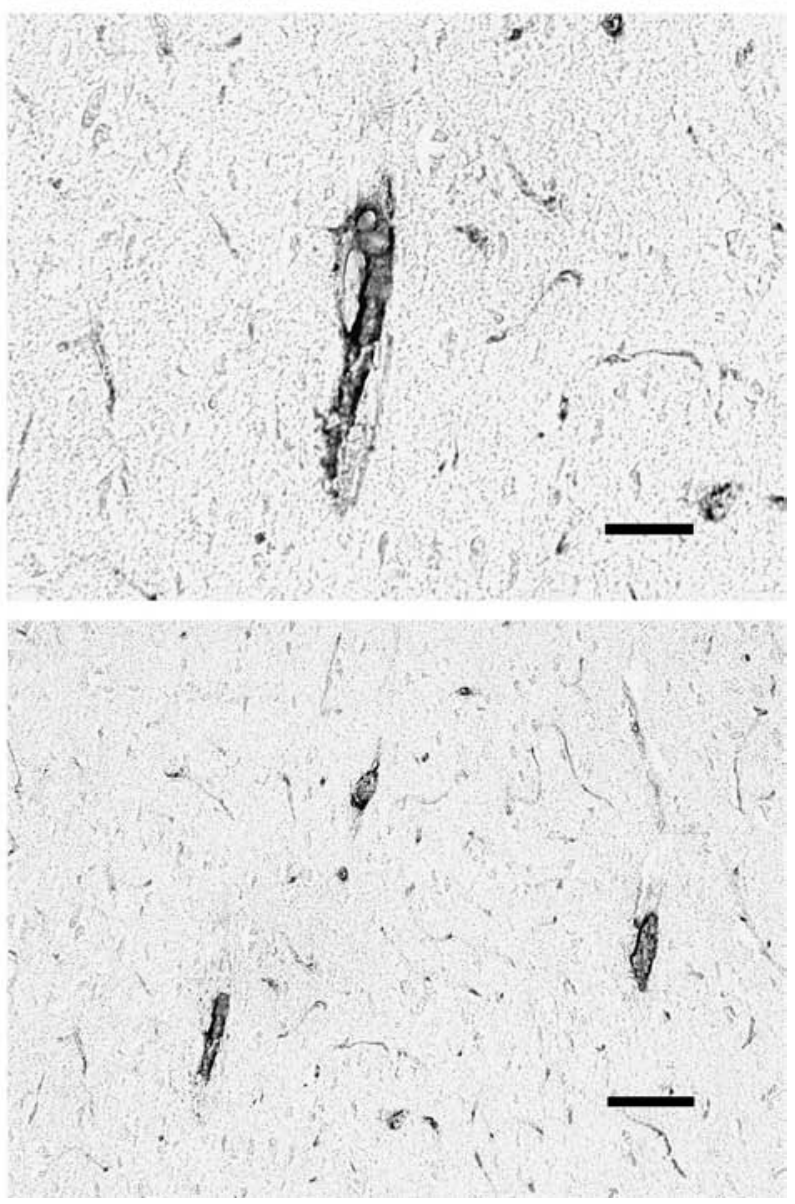

FIG. 1. Microclots $48 \mathrm{~h}$ following TBI are found in large (a), medium (b), and small (c) arterioles. Stain for clots uses an antibody to thrombin-antithrombin complexes and diaminobenzidine counterstain (see text for details) (bars $=40 \mu \mathrm{m}$ ).

In addition, the molecule has been reported to have toxic effects on the CNS, on the blood-brain barrier (BBB) (Polavarapu et al., 2007; Yepes et al., 2003), on neurons themselves, and on other elements of the neuropil (Armstead et al., 2006; Yepes and Lawrence, 2004).

To be of use in thromboprophylaxis, a fibrinolytic agent should selectively lyse potentially occlusive clots during their formation, without affecting hemostatic clots or exerting ex- travascular toxicity. However, all existing fibrinolytics are short-lived $(<30 \mathrm{~min})$ and small $(<10 \mathrm{~nm}$ diameter) agents, capable of diffusion into hemostatic clots. None can be used safely, even in patients diagnosed with a high risk of imminent or recurrent thrombosis. However, prior studies showed that coupling tPA to red blood cells (RBCs) produces longcirculating enzymatically active complexes, $\mathrm{RBC} / \mathrm{tPA}$, that neither lyse hemostatic clots nor enter the CNS. Therefore coupling tPA to RBC converts tPA into an effective and safe thromboprophylactic agent. Injection of either preformed RBC/tPA (Ganguly et al., 2006; Ganguly et al., 2007; Murciano et al., 2003) or tPA derivatives targeted to circulating RBCs (Zaitsev et al., 2006) prevents subsequent formation of occlusive venous and arterial clots, with no overt harmful effects on the carrier RBC (Ganguly et al., 2006; Ganguly et al., 2007; Murciano et al., 2003), activation of coagulation (Murciano et al., 2003), or impaired post-surgical hemostasis (Zaitsev et al., 2006). Furthermore, pretreatment with RBC/tPA provides rapid dissolution of cerebrovascular thromboemboli in a mouse model, thereby providing fast and stable reperfusion, alleviating ischemic brain injury, and eliminating lethality without the adverse side effects associated with injection of tPA (Danielyan et al., 2008).

Thus RBC/tPA has several key advantages over free tPA in the context of preventing thrombotic brain ischemia (Murciano et al., 2003), which makes it a good candidate for testing in TBI models. The purpose of this study was to evaluate and compare the effects of different forms of tPA after moderate TBI in the rat.

\section{Methods}

\section{Treatment groups}

In an effort to separate the various actions of tPA, we used two experimental treatment groups: RBC-bound wild-type tPA (RBC/tPA) and free (unbound) tPA. A third (control) group received vehicle, phosphate-buffered saline with bovine serum albumin (PBS-BSA), injection only.

The day prior to injury, $1.5 \mathrm{~mL}$ of blood was removed from each rat. All rats were assigned to one of the three groups and received the same volume of RBC transfusions to maintain hematocrits within the normal range. The preparations were readied, coded, and labeled by one researcher; all others were blinded with respect to the groups' compositions for the duration of the experiments. All animals were given a single 1.5-mL femoral vein injection of tPA or vehicle in autologous blood $15 \mathrm{~min}$ after injury.

\section{Preparation of treatments}

Human recombinant tPA (Alteplase) was obtained from Genentech (South San Francisco, CA). RBCs were isolated by centrifugation from fresh anti-coagulated rat blood. Approximately $6 \times 10^{4}$ molecules of tPA were attached per RBC as previously described (Ganguly et al., 2006; Ganguly et al., 2005; Murciano et al., 2003; Muzykantov and Murciano, 1996). The conjugation process does not affect the enzymatic activity of tPA or RBC biocompatibility (Ganguly et al., 2007). The $\mathrm{RBC} / \mathrm{tPA}$ conjugate was suspended in PBS-BSA containing $2 \mathrm{mg} / \mathrm{mL}$ BSA, and intact washed RBCs (final hematocrit $50 \%$ ) were added to all formulations in order to compensate for the potential effects of hematocrit changes in animals. 
Table 1. Microclot Density ${ }^{\mathrm{a}}$

\begin{tabular}{lccc}
\hline Group & Vehicle & RBC/tPA & Free tPA \\
\hline $\begin{array}{l}\text { No. per group } \\
\text { Peri-lesional }\end{array}$ & 4 & 4 & 5 \\
Whole hemisphere & $0.0304( \pm .0313)$ & $0.0349( \pm .0537)$ & $0.0504( \pm .0306)$ \\
Left hemisphere & & & \\
Right hemisphere & $0.0248( \pm .0227)$ & $0.0337( \pm .0474)$ & $0.0465( \pm .0318)$ \\
Whole brain & $0.0193( \pm .0156)$ & $0.0346( \pm .0411)$ & $0.0672( \pm .0835)$ \\
\hline
\end{tabular}

${ }^{\mathrm{a}}$ Mean number per $\mathrm{mm}^{2}( \pm \mathrm{SD})$.

\section{Lateral fluid percussion injury}

The procedure for the fluid percussion model of brain injury has been previously described in detail (McIntosh et al., 1989). Briefly, 65 male Sprague-Dawley rats (325-375 g) were anesthetized with sodium pentobarbital $(60 \mathrm{mg} / \mathrm{kg}$ IP) and a 5 -mm craniotomy was performed over the left cortex midway between the lambda and the bregma and $4 \mathrm{~mm}$ lateral to the sagittal suture. With the dura intact, a female Luer-Lok fitting was secured in the craniotomy site. The animals were placed on heating pads until the time of injury. Ninety minutes after anesthesia induction, the animals were attached to the fluid percussion device via the Luer-Lok fitting and injured at a moderate severity level (2.4-2.6 atm).

All animals were pair-housed with a 12-h light/dark cycle with access to food and water ad libitum, and food intake and animal weight were measured every 2 days. Twelve animals $(28 \%)$ died, presumably of their injuries, prior to sacrifice. Since they were evenly distributed among treatment groups, they were excluded from further analysis. All experiments were carried out in accordance with policies set forth by the University of Pennsylvania Institutional Animal Care and Use Committee, and in accordance with NIH guidelines for the humane and ethical treatment of animals.

\section{Histological preparation}

Sacrifice was at 2 (for assessment of intravascular microthrombosis and blood-brain barrier integrity), or at 7 days (for assessment of lesion volume and hippocampal cell loss). All animals were given an overdose of sodium pentobarbital $(200 \mathrm{mg} / \mathrm{kg})$ and transcardially perfused with heparinized saline followed by $4 \%$ paraformaldehyde. The brains were removed, post-fixed overnight in $4 \%$ paraformaldehyde, placed in PBS buffer for 3 days, and immersed in 30\% sucrose until saturated (4-5 days). The brains were then flash frozen in isopentane at $-30^{\circ} \mathrm{C}$ to $-40^{\circ} \mathrm{C}$ and sectioned coronally. Twenty-micron sections ( $3 \mathrm{~mm}$ behind the frontal poles to the cerebellum) were cut on a rotary microtome, and sections $300 \mu \mathrm{m}$ apart were saved for analysis.

Tissue sections for IgG immunostaining were incubated in PBS, anti-rat IgG (1:200; Vector Laboratories, Burlingame, CA), then incubated with anti-sheep IgG (1:400) secondary antibody, and 3,3'-diaminobenzidine tetrahydrochloride (Vector Laboratories) for visualization (Hoshino et al., 1996). Tissues for intravascular microthrombi assessment were incubated overnight with a 1:300 dilution of a sheep polyclonal antibody used to recognize both free antithrombin III, and antithrombin III in antithrombin III-protease complexes (American Diagnostica, Stamford, CT). The sections were
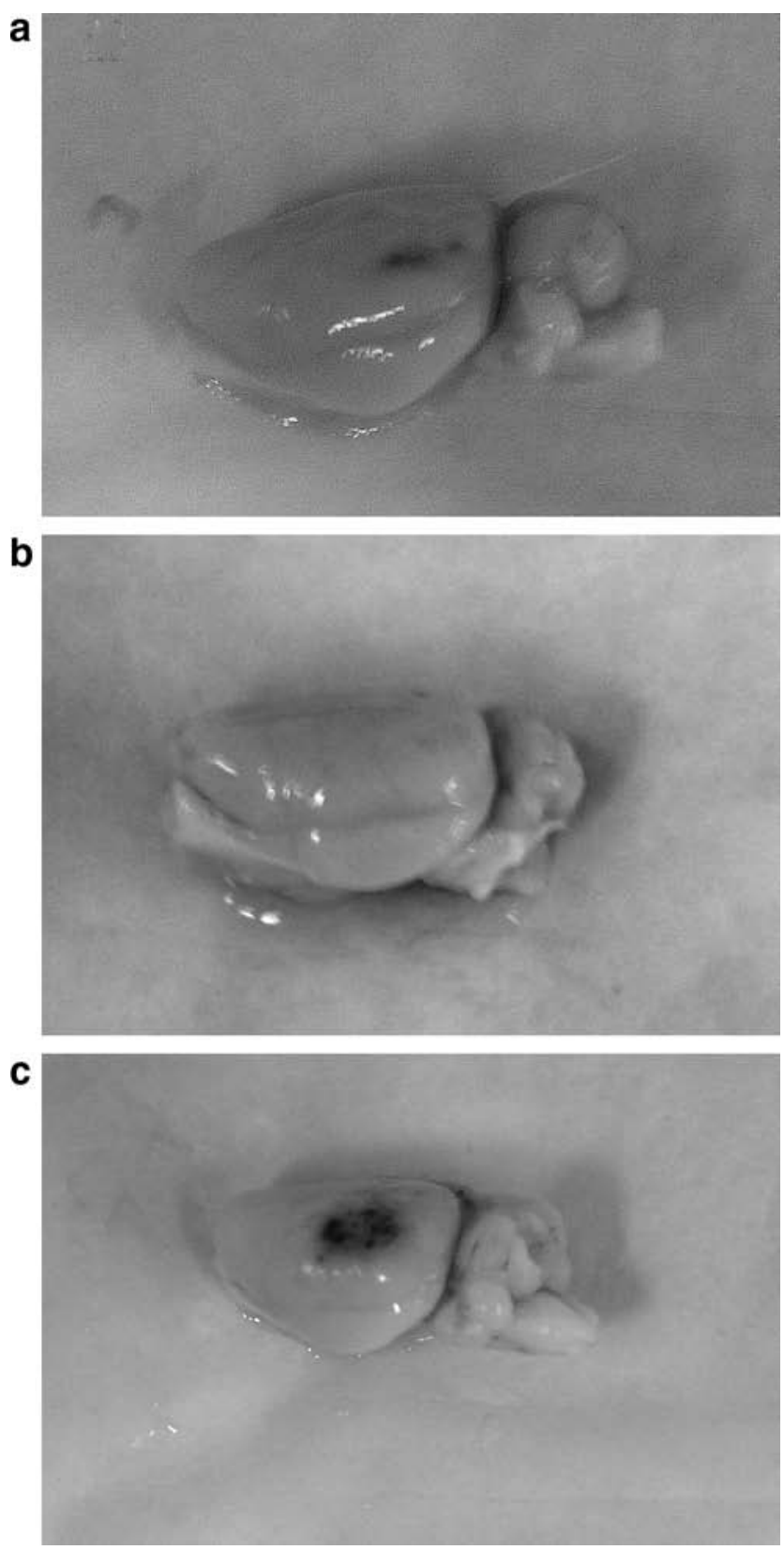

FIG. 2. Amount of post-traumatic hemorrhage. Photographs of the impact site taken at $48 \mathrm{~h}$ after TBI in representative animals (a, vehicle; $\mathbf{b}, \mathrm{RBC} / \mathrm{tPA}$; $\mathbf{c}$, free tPA). 
a

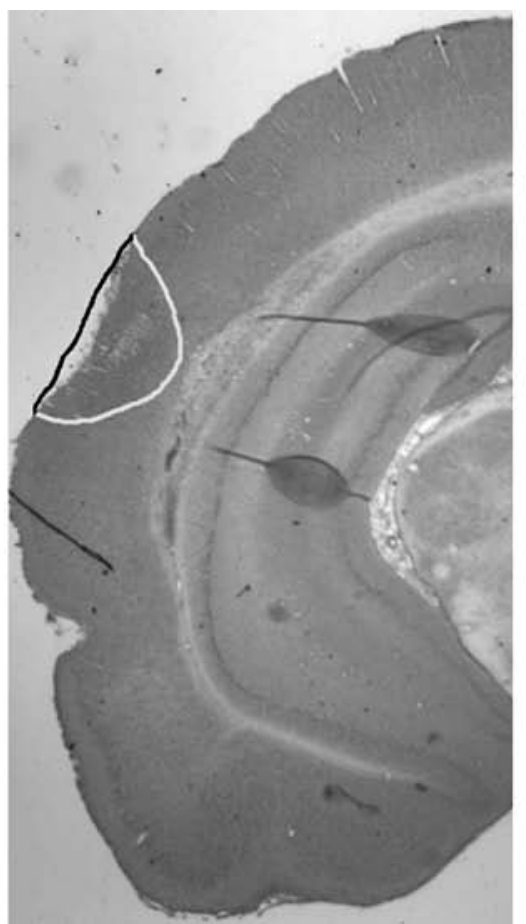

b

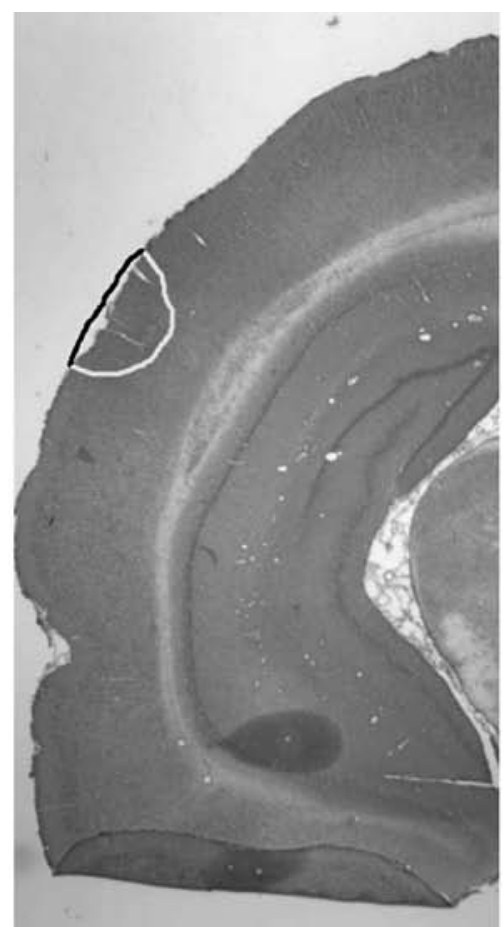

C

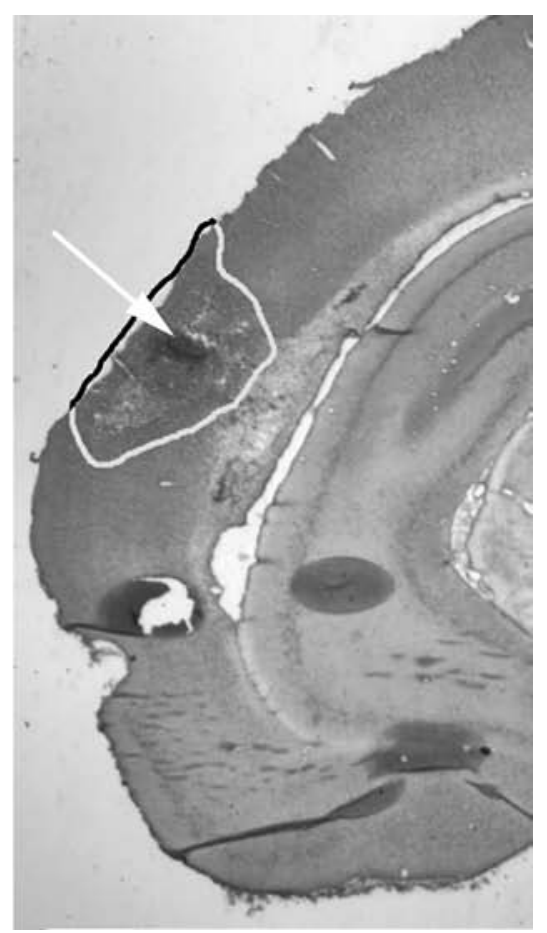

FIG. 3. Cortical tissue loss. Representative H\&E-stained slices of injured brain. Tissue loss (cavitation, hemorrhage, and neuronal necrosis) is outlined, and the arrow points to an area of hemorrhage (a, vehicle; $\mathbf{b}, \mathrm{RBC} / \mathrm{tPA}$; $\mathbf{c}$, free tPA).

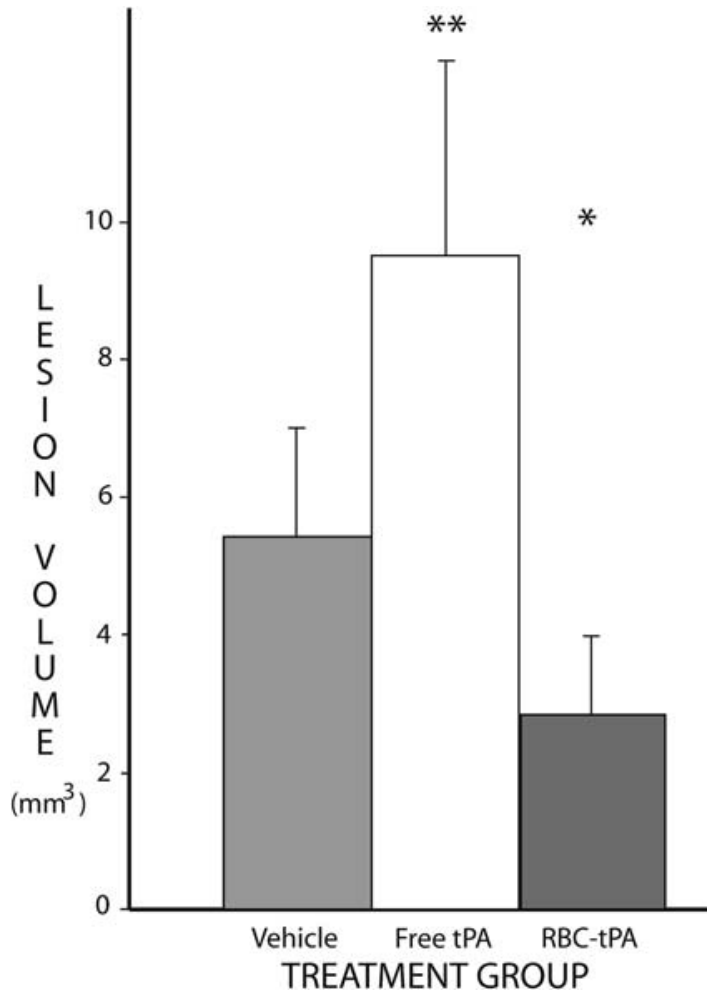

FIG. 4. Volume of cortical tissue loss. The mean total volume loss for each of the three treatment groups is shown. The vertical bars represent standard deviations. Tissue loss was significantly lower with RBC-bound tPA $\left({ }^{*}\right)$, and significantly higher with free tPA $\left({ }^{* *}\right)$ than with vehicle. then incubated with anti-sheep IgG (1:400) secondary antibody, and 3,3'-diaminobenzidine tetrahydrochloride (Vector Laboratories) for visualization. Staining for evaluation of cortical tissue loss was with hematoxylin and eosin (H\&E), and that for evaluation of hippocampal tissue loss employed cresyl violet Nissl stain.

\section{Evaluation of intravascular microthrombi}

The total number of microclots were counted in each section of each specimen. To estimate the density of microclots, the surface area of each microscopic specimen was computed from the digital images, using MCID Imaging software (Interfocus Imaging, Cambridge, England), and the number of microclots per square millimeter (microclot burden) was calculated for each hemisphere of each section (Stein et al., 2002). Treatment groups were compared with respect to microclot burdens.

\section{Evaluation of blood-brain barrier integrity}

Endogenous $\mathrm{IgG}$ is not normally seen in the rat brain in any quantity; its presence is an indication of extravasation secondary to BBB breakdown. All sections were examined by light microscopy. Areas containing IgG staining were outlined and measured, and the total volume of extravasation was calculated using the same formula as for cortical tissue loss as described below.

\section{Evaluation of cortical and hippocampal tissue loss}

For cortical tissue, the area of each lesion was outlined. Where tissue had been lost at the cortical surface, the surface 
line was reconstructed by referring to the opposite hemisphere. Areas of neuronal necrosis, cavitation, and hemorrhage were included within the lesion outline. The area of cortical tissue loss in each section was measured using NIH Image Software (v1.62). All sections containing identifiable tissue loss were included. The total volume of tissue loss was obtained using the formula:

$$
\begin{aligned}
& \Sigma\left[\left(\text { Area Lesion }_{n}+\text { Area Lesion }_{n+1}\right) / 2\right] \\
& \quad \times(\text { Distance between Sections } n \text { and } n+1)
\end{aligned}
$$

For each animal, five nonadjacent sections were chosen from -3.3 to -3.8 , and four nonadjacent sections from -5.3 to -5.6 bregma for evaluation of hippocampal tissue loss. The CA3 subfield of the hippocampus was centered on the image field and a precalibrated length of $1 \mathrm{~mm}$ along the region previously characterized as the site of maximal damage in the ipsilateral pyramidal layer was chosen for counting neurons. Viable neurons, which had well-defined cell bodies and nuclei, were counted in the selected area. For each slide, the corresponding cell count on the uninjured side was recorded. The percentage of cell loss in each section was obtained using the formula: $100 \% \times\left(\mathrm{N}_{\text {control }}-\mathrm{N}_{\text {injured }}\right) / \mathrm{N}_{\text {control }}$. A total count of neuron loss for each animal was obtained by averaging the counts from the individual sections (Leoni et al., 2000).

\section{Statistical analysis}

Comparisons among the different groups were performed using a one-way analysis of variance (ANOVA), followed by post-hoc Bonferroni tests for individual comparisons (Stata 9; StataCorp, College Station, TX). Statistical significance was set at $p<0.05$.

\section{Results}

The means and standard deviations of microclot burden calculations (number of microthrombi/tissue area) are shown in Table 1. The burden was calculated for the peri-lesional area

b

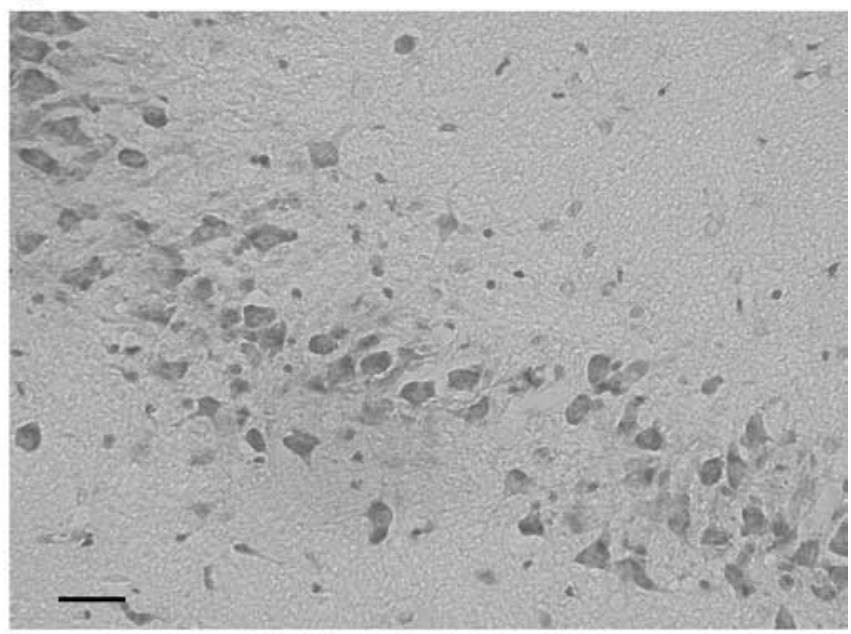

d

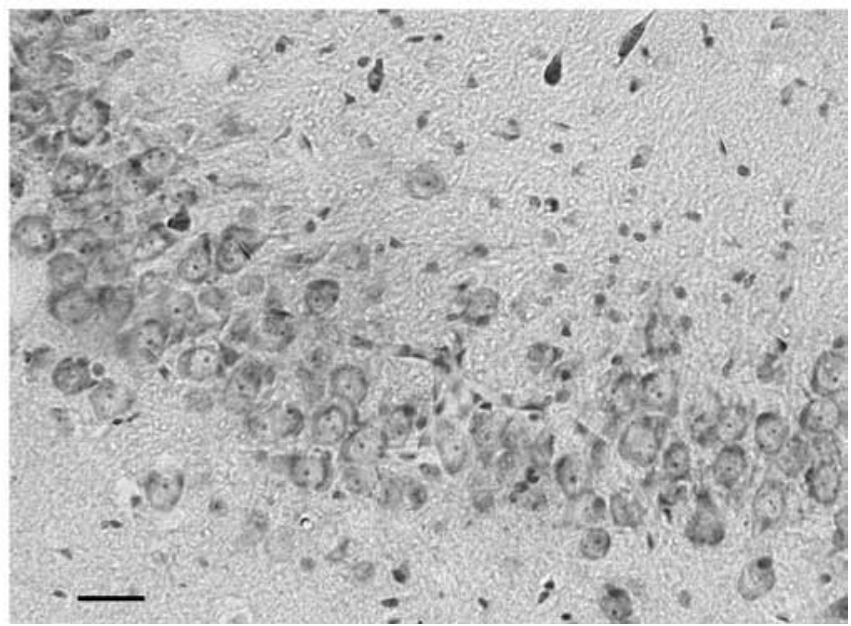

FIG. 5. Hippocampal CA3 cell density. Representative Nissl-stained coronal sections from the treatment groups. (a) Image from a normal, uninjured animal, showing many healthy neurons with pale, plump nuclei and prominent nucleoli. (b) Image from a vehicle-treated animal, displaying striking neuronal loss. (c) Image from a free tPA-treated animal, showing intermediate neuronal loss. (d) Image from an RBC/tPA-treated animal, showing only modest neuronal loss (original magnification $20 \mathrm{x} \times$; bar $=50 \mu \mathrm{m}$ ). 
of the left hemisphere, the entire left hemisphere, and the entire brain. No differences were found among the three treatment groups in any of these calculations $(p=0.775)$. The mean volume of IgG staining was $27.6( \pm 11.6) \mathrm{mm}^{3}$ in the vehicle group $(\mathrm{n}=4), 33.8( \pm 23.4) \mathrm{mm}^{3}$ in the RBC/tPA group $(\mathrm{n}=4)$, and $30.5( \pm 15.2) \mathrm{mm}^{3}$ in the free tPA group $(\mathrm{n}=5)$, suggesting no significant difference with regard to BBB breakdown $(p=.609)$.

Both gross and microscopic inspection suggested that, compared to vehicle, rats treated with free tPA had more cortical hemorrhage, whereas RBC/tPA-treated rats appeared to have less (Fig. 2).

At 7 days after TBI, significant differences in outcome were noted among the treatment groups. The mean lesion volumes were $5.44 \mathrm{~mm}^{3}( \pm 1.55)$ in the 9 control rats administered vehicle. The mean lesion volumes for the rats treated with $\mathrm{RBC} / \mathrm{tPA}(\mathrm{n}=6)$ and free tPA $(\mathrm{n}=9)$ were $3.03( \pm 1.24)$ and $9.56( \pm 2.44)$, respectively. These differences, illustrated in Figures 3 and 4, are significant, both for ANOVA ( $p=0.006)$, and for between-group comparisons. The lesion volumes in rats treated with $\mathrm{RBC} / \mathrm{tPA}$ were significantly smaller $(p<0.01)$ than in all other treatment groups, whereas they were significantly larger $(p<0.05)$ with free wild-type tPA. In addition, hippocampal cell losses were $50.9 \%( \pm 20.9 \%)$ in the 8 vehicle-treated rats, $43.2 \%( \pm 15.7 \%)$ in the 8 treated with free $\mathrm{tPA}$, and $17.9 \%( \pm 8.3 \%)$ in the $4 \mathrm{RBC} / \mathrm{tPA}$-treated rats (Figs. 5 and 6). These differences were significant $(p=0.007)$ for one-way ANOVA. The RBC/tPA group showed significantly less $(p<0.01)$ cell loss than the other two groups; between-group comparisons were not significant for the free tPA or control groups.

\section{Discussion}

The present results reveal a neuroprotective effect of $\mathrm{RBC} / \mathrm{tPA}$ in rats injured by lateral fluid percussion. There was significantly less cortical and hippocampal damage compared to animals treated with vehicle or with free tPA. No hemorrhagic complications have been observed in rats treated with $\mathrm{RBC} / \mathrm{tPA}$, which confirms its unprecedented safety and potential utility in the cerebral vasculature, even shortly after traumatic injury.

The therapeutic potency of RBC/tPA may be related to its long biological half-life, which is in contrast to the short-lived yet relatively indiscriminate lytic activity of free tPA. This latter behavior is reflected by the greater apparent cortical hemorrhage associated with free tPA. In contrast to free tPA, $\mathrm{RBC} / \mathrm{tPA}$ is confined to the bloodstream, does not attack hemostatic mural plugs, and due to the partial masking effect of the RBC glycocalyx on tPA (Ganguly et al., 2007), is more strictly dependent on positive regulation by fibrin in activation of plasminogen (Ganguly et al., 2006).

We were unable to quantify the amount of bleeding in this study. Traumatic hemorrhage takes many forms (microscopic, contusional, subdural, and parenchymal hematomas). Discrete hematomas have a tendency to fragment and separate from tissue during processing. Hence we can do no more than suggest a difference in bleeding tendency among the three groups.

We were also unable to definitively address the mechanisms by which $\mathrm{RBC} / \mathrm{tPA}$ exerted its protective effect.

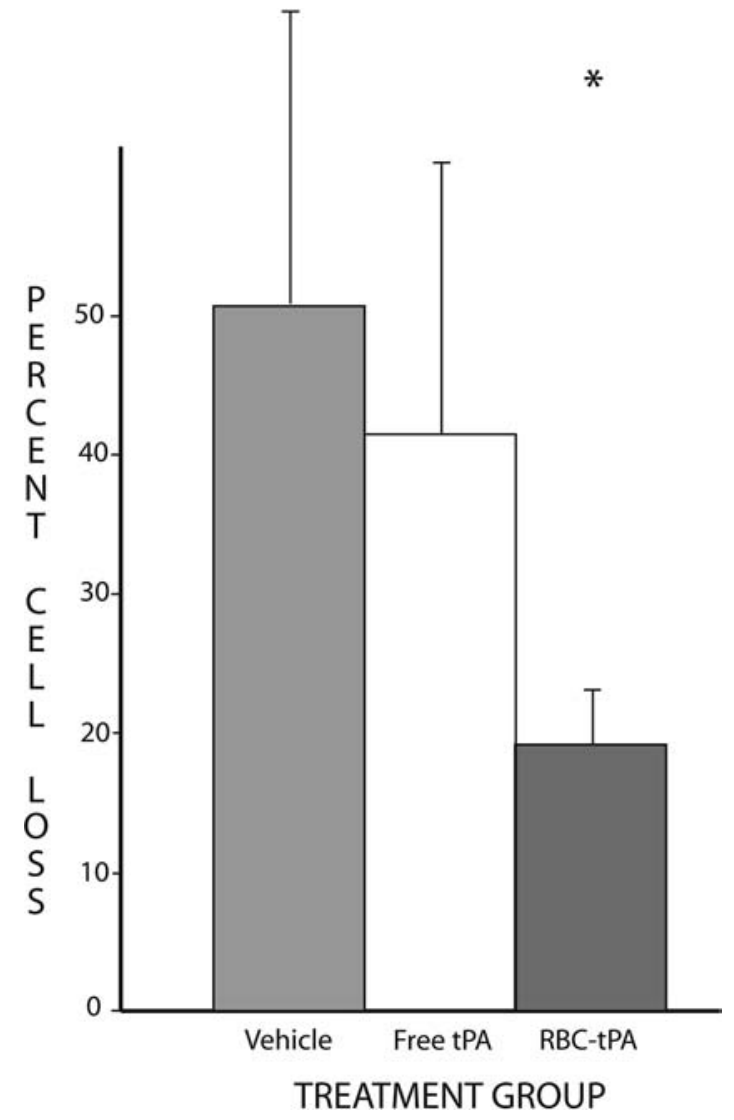

FIG. 6. Percentage of hippocampal cell loss. Cell counts in the defined areas of CA3 were compared on the injured (left) and uninjured (right) side of each slice studied. The mean percentage loss for each of the three treatment groups is shown. The vertical bars represent standard deviations. Cell loss was significantly lower in the $\mathrm{RBC} / \mathrm{tPA}$ group $\left(^{*}\right)$.

Possible mechanisms and sites of RBC/tPA action include dissolution of secondary clots and initiation of signaling pathways within the cerebral vasculature. In support of the latter notion, a recent study revealed that $\mathrm{RBC} / \mathrm{tPA}$ exerts anti-inflammatory (MAPK inhibition) and neuroprotective effects in models of cerebrovascular pathology in the pig, whereas free tPA augmented pro-inflammatory signaling via lipoprotein-receptor protein and MAPK activation, and this aggravated CNS injury (Armstead et al., 2008). These data indicate that $\mathrm{RBC} / \mathrm{tPA}$ may exert an additional mechanism of neuroptotection in the TBI by affecting pathological signaling in the CNS. The precise nature of the non-fibrinolytic protective and the injurious signaling seen with RBC/tPA and tPA, respectively, are currently not fully understood, but may be explained by their accessibility to different intravascular and parenchymal subsets of tPA receptors.

Our inability to demonstrate an effect on microclot burden does not exclude the possibility of a thrombolytic effect of $\mathrm{RBC} / \mathrm{tPA}$. Treatment following TBI was carried out within minutes, whereas the microclot burden was assessed at $48 \mathrm{~h}$. Experimental microemboli in rodents are lysed within hours (Bdeir et al., 2000), so it is likely that the 
microclots seen at $48 \mathrm{~h}$ were formed after the $\mathrm{RBC} / \mathrm{tPA}$ had lost much of its potency. If early microclots were lysed by $\mathrm{RBC} / \mathrm{tPA}$ before they had an opportunity to cause permanent ischemic injury, the effects might not be seen at $48 \mathrm{~h}$. Additional experiments are needed to determine whether the benefit of $\mathrm{RBC} / \mathrm{tPA}$ is due to its thrombolytic activity, or is related to some other proteolytic or non-proteolytic effects.

\section{Acknowledgments}

This study was supported in part by U.S. Public Health Service grants from the National Institutes of Health (RO1sHL077760-01A2, HL090697, HL76406, CA83121, HL76206, HL07971, HL81864, and NS053410), and the University of Pennsylvania Institute for Translational Medicine and Therapeutics (D.B.C.).

This material was presented in part before the International Neurotrauma Society in Rotterdam, The Netherlands in 2006.

\section{Author Disclosure Statement}

No conflicting financial interests exist.

\section{References}

Armstead, W.M., Ganguly, K., Kiessling, J.W., Higazi, A., Zaitsev, S., Cines, D., Bdeir, K., and Muzykantov, V.R. (2008). Rbc-Tpa helps prevent hypercapnic and hypotensive cerebrovascular impairment by inhibiting Erk Mapk upregulation after cerebral hypoxia/ischemia. Stroke 30, 165 [abstract].

Armstead, W.M., Nassar, T., Akkawi, S., Smith, D.H., Chen, X.H., Cines, D.B., and Higazi, A.A. (2006). Neutralizing the neurotoxic effects of exogenous and endogenous tPA. Nat. Neurosci. 9, 1150-1155.

Bdeir, K., Murciano, J.C., Tomaszewski, J., Koniaris, L., Martinez, J., Cines, D.B., Muzykantov, V.R., and Higazi, A.A. (2000). Urokinase mediates fibrinolysis in the pulmonary microvasculature. Blood 96, 1820-1826.

Danielyan, K., Ganguly, K., Ding, B.S., Atochin, D., Zaitsev, S., Murciano, J.C., Huang, P.L., Kasner, S.E., Cines, D.B., Muzykantov, V.R. (2008). Cerebrovascular thromboprophylaxis in mice by erythrocyte-coupled tissue-type plasminogen activator. Circulation 118, 1442-1449.

Fleck, R.A., Rao, L.V., Rapaport, S.I., and Varki, N. (1990). Localization of human tissue factor antigen by immunostaining with monospecific, polyclonal anti-human tissue factor antibody. Thromb. Res. 59, 421-437.

Ganguly, K., Goel, M.S., Krasik, T., Bdeir, K., Diamond, S.L., Cines, D.B., Muzykantov, V.R., and Murciano, J.C. (2006). Fibrin affinity of erythrocyte-coupled tissue-type plasminogen activators endures hemodynamic forces and enhances fibrinolysis in vivo. J. Pharmacol. Exp. Ther. 316, 11301136.

Ganguly, K., Krasik, T., Medinilla, S., Bdeir, K., Cines, D.B., Muzykantov, V.R., and Murciano, J.C. (2005). Blood clearance and activity of erythrocyte-coupled fibrinolytics. J. Pharmacol. Exp. Ther. 312, 1106-1113.

Ganguly, K., Murciano, J.C., Westrick, R., Leferovich, J., Cines, D.B., and Muzykantov, V.R. (2007). The glycocalyx protects erythrocyte-bound tissue-type plasminogen activator from enzymatic inhibition. J. Pharmacol. Exp. Ther. 321, 158-164.
Graham, D.I., and Adams, J.H. (1971). Ischaemic brain damage in fatal head injuries. Lancet 1, 265-266.

Graham, D.I., Ford, I., Adams, J.H., Doyle, D., Teasdale, G.M., Lawrence, A.E., and McLellan, D.R. (1989) Ischaemic brain damage is still common in fatal non-missile head injury. J. Neurol. Neurosurg. Psychiatry 52, 346-350.

Hlatky, R., Contant, C.F., Diaz-Marchan, P., Valadka, A.B., and Robertson, C.S. (2004). Significance of a reduced cerebral blood flow during the first 12 hours after traumatic brain injury. Neurocrit. Care 1, 69-83.

Hoshino, S., Kobayashi, S., and Nakazawa, S. (1996). Prolonged and extensive IgG immunoreactivity after severe fluidpercussion injury in rat brain. Brain Res. 711, 73-83.

Leoni, M.J., Chen, X.H., Mueller, A.L., Cheney, J., McIntosh, T.K., and Smith, D.H. (2000). Nps 1506 attenuates cognitive dysfunction and hippocampal neuron death following brain trauma in the rat. Exp. Neurol. 166, 442-449.

Lu, D., Mahmood, A., Goussev, A., Schallert, T., Qu, C., Zhang, Z.G., Li, Y., Lu, M., and Chopp, M. (2004). Atorvastatin reduction of intravascular thrombosis, increase in cerebral microvascular patency and integrity, and enhancement of spatial learning in rats subjected to traumatic brain injury. J. Neurosurg. 101, 813-821.

McIntosh, T.K., Vink, R., Noble, L., Yamakami, I., Fernyak, S., Soares, H., and Faden, A.L. (1989). Traumatic brain injury in the rat: Characterization of a lateral fluid- percussion model. Neuroscience 28, 233-244.

Murciano, J.C., Medinilla, S., Eslin, D., Atochina, E., Cines, D.B., and Muzykantov, V.R. (2003). Prophylactic fibrinolysis through selective dissolution of nascent clots by Tpa-carrying erythrocytes. Nat. Biotechnol. 21, 891-896.

Muzykantov, V.R., and Murciano, J.C. (1996). Attachment of antibody to biotinylated red blood cells: Immuno-red blood cells display high affinity to immobilized antigen and normal biodistribution in rats. Biotechnol. Appl. Biochem. 24, 41-45.

Narita, M., Bu, G., Herz, J., and Schwartz, A.L. (1995). Two receptor systems are involved in the plasma clearance of tissuetype plasminogen activator (T-Pa) in vivo. J. Clin. Invest. 96, 1164-1168.

Polavarapu, R., Gongora, M.C., Yi, H., Ranganthan, S., Lawrence, D.A., Strickland, D., and Yepes, M. (2007). Tissue-type plasminogen activator-mediated shedding of astrocytic lowdensity lipoprotein receptor-related protein increases the permeability of the neurovascular unit. Blood 109, 32703278.

Stein, S.C., Chen, X.-H., Sinson, G.P., Smith, D.H. (2002). Intravascular coagulation: A major secondary insult in nonfatal traumatic brain injury. J. Neurosurg. 97, 1373-1377.

Stein, S.C., Graham, D.I., Chen, X.H., and Smith, D.H. (2004). Association between intravascular microthrombosis and cerebral ischemia in traumatic brain injury. Neurosurgery 54, 687691; discussion 691.

Stein, S.C., and Smith, D.H. (2004). Coagulopathy in traumatic brain injury. Neurocritical Care 1, 479-488.

Stiefel, M.F., Udoetuk, J.D., Spiotta, A.M., Gracias, V., Goldberg, A., Maloney-Wilensky, E., Bloom, S., and Le Roux, P.D. (2006). Conventional neurocritical care and cerebral oxygenation after traumatic brain injury. J. Neurosurg. 105, 568-575.

Teasdale, G.M., and Graham, D.I. (1998). Craniocerebral trauma: Protection and retrieval of the neuronal population after injury. Neurosurgery 43, 723-737.

Yepes, M., and Lawrence, D.A. (2004). New functions for an old enzyme: Nonhemostatic roles for tissue-type plasminogen 
activator in the central nervous system. Exp. Biol. Med. (Maywood) 229, 1097-1104.

Yepes, M., Sandkvist, M., Moore, E.G., Bugge, T.H., Strickland, D.K., and Lawrence, D.A. (2003). Tissue-type plasminogen activator induces opening of the blood-brain barrier via the LDL receptor-related protein. J Clin. Invest. 112, 15331540 .

Zaitsev, S., Danielyan, K., Murciano, J.C., Ganguly, K., Krasik, T., Taylor, R.P., Pincus, S., Jones, S., Cines, D.B., and Muzykantov, V. (2006). Human complement receptor type 1directed loading of tissue plasminogen activator on circulating erythrocytes for prophylactic fibrinolysis. Blood 108, 18951902.

Address correspondence to: Sherman C. Stein, M.D. Department of Neurosurgery University of Pennsylvania School of Medicine 310 Spruce Street Philadelphia, PA 19106

E-mail: sherman.stein@uphs.upenn.edu 